\title{
Managing Functional Neurological Disorders: Protocol of a Cohort Study on Psychogenic Non-Epileptic Seizures Study
}

This article was published in the following Dove Press journal:

Neuropsychiatric Disease and Treatment

\author{
Hamada Hamid Altalib ${ }^{1,2}$ \\ Daniela Galluzzo iD ${ }^{3}$ \\ Stephanie Argraves ${ }^{1,4}$ \\ Joseph Goulet (iD) ${ }^{1,5}$ \\ Yarden Bornovski ${ }^{1,2}$ \\ Kei-Hoi Cheung (iD) ${ }^{1,5}$ \\ Ebony Jackson-Shaheed ${ }^{1,2}$ \\ Benjamin Tolchin (D) ${ }^{1,2}$ \\ Brenda T Fenton' \\ Mary Jo Pugh (iD) 6,7 \\ 'VA Connecticut Healthcare System, \\ West Haven, CT, USA; ${ }^{2}$ Comprehensive \\ Epilepsy Center, Department of \\ Neurology, Yale School of Medicine, New \\ Haven, CT, USA; ${ }^{3}$ Westchester Medical \\ Center, Westchester, NY, USA; \\ ${ }^{4}$ Department of Psychiatry, Yale School of \\ Medicine, New Haven, CT, USA; \\ ${ }^{5}$ Department of Emergency Medicine, \\ Yale School of Medicine, New Haven, CT, \\ USA; ${ }^{6}$ University of Utah, Salt Lake City, \\ UT, USA; ${ }^{7}$ Salt Lake City Veterans \\ Healthcare System, Salt Lake City, \\ UT, USA
}

Correspondence: Hamada Hamid Altalib VA Connecticut Healthcare System, West Haven, CT, USA

Email hamada.hamid@yale.edu
Background: Functional neurological disorders (FNDs) are neurological symptoms that cannot be explained by an underlying neurological lesion or other medical illness and that do not have clear neuropathological correlates. Psychogenic non-epileptic seizures (PNES) are a common and highly disabling form of FND, characterized by paroxysmal episodes of involuntary movements and altered consciousness that can appear clinically similar to epileptic seizures. PNES are unique among FNDs in that they are diagnosed by video electroencephalographic (VEEG), a well-established biomarker for the disorder. The course of illness and response to treatment of PNES remain controversial. This study aims to describe the epidemiology of PNES in the Department of Veterans Affairs Healthcare System (VA), evaluate outcomes of veterans offered different treatments, and compare models of care for PNES.

Methods: This electronic health record (EHR) cohort study utilizes an informatics search tool and a natural language processing algorithm to identify cases of PNES nationally. We will use VA inpatient, outpatient, pharmacy, and chart abstraction data across all 170 medical centers to identify cases in fiscal years 2002-2018. Outcome measurements such as seizure frequency, emergency room visits, hospital admissions, suicide-related behavior, and the utilization of psychotherapy prior to and after PNES diagnosis will be used to assess the effectiveness of models of care.

Discussion: This study will describe the risk factors and course of treatment of a large cohort of people with PNES. Since PNES are cared for by a variety of different modalities, treatment orientations, and models of care, effectiveness outcomes such as seizure outcomes and utilization of emergency visits for seizures will be assessed. Outcome measurements such as seizure frequency, emergency room visits, hospital admissions, suicide-related behavior, and psychotherapy prior to and after PNES diagnosis will be used to assess the effectiveness of models of care.

Keywords: Veterans Administration, VA, electronic health record, EHR, psychogenic nonepileptic seizures, PNES, functional neurological disorders, FND

\section{Contributions to the Literature}

- Previous studies have argued for an integrated mental health model. We are exploring the effectiveness of different models in managing functional neurological disorders, which requires coordination between mental health, primary care, and neurology.

- Several small studies have demonstrated the efficacy of different modalities of psychotherapy for the management of PNES. We will explore the effectiveness of different approaches for the management of PNES. 
- PTSD has been described as a major risk factor for PNES. Our cohort analysis will explore whether treatment of PTSD decreases the incidence of PNES in veterans at risk for developing the condition.

\section{Background}

\section{The Challenges of Identifying Functional Neurological Disorders}

Functional neurological disorders (FNDs), formerly known as conversion disorders, are characterized by preoccupation of neurological symptoms that are driven by a variety of psychopathology. The differential diagnosis of FNDs is wide and covers the full range of neurologic disease, leading to a protracted, expensive diagnostic workup. ${ }^{1}$ Psychogenic nonepileptic seizures (PNES), a relatively common and highly disabling form of FND, consist of paroxysmal episodes of altered consciousness and/or involuntary movements that can appear clinically similar to epileptic seizures. ${ }^{2-4}$ PNES are unique among FNDs in that they are diagnosed through the biomarker of video-electroencephalogram (VEEG). ${ }^{5}$ While new structural and functional neuroimaging studies characterize the dysfunctional networks involved in PNES, VEEG remains the gold standard for diagnosis. ${ }^{6}$ Seizure-like events are diagnosed by capturing all habitual episodes on VEEG, with normal awake brain activity without epileptiform abnormalities immediately before, during, and following each episode. VEEG monitoring is usually performed in an inpatient setting to allow safe titration off anti-epileptic drugs while minimizing risk of self-injury from violent convulsions, falls, or potential epileptic seizures. ${ }^{5}$ However, people with infrequent events (such as less than once a week) may present a diagnostic challenge since an observable attack may not occur during a VEEG session.

Since epilepsy is primarily diagnosed clinically and PNES events can be very difficult to distinguish from epileptic seizures, people with PNES are usually misdiagnosed with epilepsy and treated ineffectively for multiple years before the appropriate diagnosis and treatment are offered. ${ }^{3,5}$ As with other FNDs, the potential for iatrogenic harm and unnecessary health-care utilization in PNES is high. For instance, people experiencing prolonged seizurelike events are often misdiagnosed as being in status epilepticus and are unnecessarily intubated and administered high dose benzodiazepines. People with PNES frequently visit emergency rooms, frequently are admitted to hospitals for seizure management, and are inappropriately started and maintained on multiple anti-seizure medications for many years. ${ }^{5,7}$

While PNES should be diagnosed with VEEG when possible, the test is not always available or diagnostic (if no seizure episode is captured on VEEG). Therefore, different levels of diagnostic certainty in PNES have been developed, not all requiring VEEG: ${ }^{8}$

- A diagnosis of documented PNES involves the recording of a seizure event during VEEG, which displays no epileptiform activity immediately before, during, or after one of the patient's typical events.

- A diagnosis of probable PNES involves either a video or direct witness of the seizures by a physician trained in PNES, along with an interictal EEG displaying no epileptiform abnormality.

- A diagnosis of possible PNES entails the description of the events by the patient, or a witness, in addition to an interictal EEG without epileptiform abnormality.

Most studies of PNES only include people who had a VEEG-confirmed diagnosis of PNES, which biases the literature to represent more severe cases in which the seizures were frequent enough to capture during an inpatient VEEG. By including probable PNES, a more generalizable sample may be studied.

\section{Study Risk Factors and Protective Factors of PNES Using VA Data}

Multiple risk factors have been identified in PNES including: sexual abuse, physical abuse or neglect, traumatic brain injury, and psychiatric comorbidities. Conditions such as post-traumatic stress disorder (PTSD), depression, and anxiety disorders have been reported at high rates. ${ }^{9}$ Some argue that PNES are essentially another phenotype or expression of PTSD or complex trauma. ${ }^{10,11}$ The course of illness of PNES is difficult to study because of the heterogeneity of PNES, the complex relationship between multiple risk factors (including moderator and mediator effects), and the requirement to study the relationships in a longitudinal model. Conducting a prospective study of a rare disease such as PNES, that requires substantial resources to establish the diagnosis, is not often feasible.

The EHR-based cohort method has been used to study many conditions and offers a model to examine rare conditions longitudinally. ${ }^{12}$ In particular, mental health and medical treatment prior to the onset of PNES may be 
examined using the cohort method. Given the longitudinal nature of the data, statistical models can be used to examine relationships of risk factors. For example (Figure 1), traumatic brain injury (TBI), PTSD, abuse history, generalized anxiety disorder, and depression may affect the development of PNES. Some factors may be confounded and not be significantly involved in the development of PNES. A longitudinal design and appropriate statistical analysis, such as path analysis, can help delineate the direct and indirect effects on the development of PNES and separate risk factors from confounders.

\section{Coordination of Care}

Consensus on the appropriate therapy for PNES has not been definitively established, due to a lack of well-powered randomized controlled trials. ${ }^{13-15}$ Proposed management has been described in phases. ${ }^{4,5}$ The first phase involves the definitive diagnosis and appropriate communication of the diagnosis, ideally by a neurologist and a psychiatrist. Clear and direct communication with nonjudgmental explanation of the diagnosis is associated with better outcomes. ${ }^{5}$ Furthermore, incorporating motivational interviewing techniques at the time of diagnosis increases the likelihood of positive psychiatric outcomes, improvement of seizure control, and quality of life. ${ }^{16}$ The second phase of management involves offering psychotherapy, and the following modalities have been recommended: cognitive behavioral therapy (CBT), prolonged exposure (PE) therapy, hypnosis, acceptance and commitment therapy (ACT), mindfulness, and psychodynamic therapy. ${ }^{15,17-21}$ To date there has only been one multi-center randomized control trial, which compared usual treatment to a manualized CBT-informed therapy, with and without the addition of a selective serotonin receptor inhibitor (SSRI). ${ }^{13} \mathrm{~A}$ modest benefit has been shown with CBT-informed therapy. ${ }^{22}$ A large multi-site randomized controlled trial of traditional CBT has completed enrollment and treatment, and results are forthcoming. ${ }^{23}$ The last phase of management includes maintenance, which requires a dedicated team to continue to follow the patient, to prevent relapse and miscommunication of the diagnosis with other health-care providers.

Coordination of care between neurologists, psychiatrists, and primary care physicians for PNES and other FNDs is critical to avoid miscommunication, redundancy in diagnostic testing and management, and loss to followup. Models of care coordination and the role of each team member varies (Figure 2). The 170 VA medical centers, including the 16 Epilepsy Centers of Excellence, use many different models to coordinate mental health-care services. The VA experience provides a natural setting to study the effectiveness of mental health coordination of care models for PNES and may have implications for caring for FND more generally.

Many neurologists, especially epilepsy specialists, prefer partnering with a designated mental health provider who works within their center and has experience and expertise with PNES $^{24}$ (Figure 2A). For instance, several VA epilepsy programs have hired mental health providers to provide direct care for veterans with epilepsy and PNES. The dominant model for delivering mental health care generally, supported by the World Health Organization (WHO), the US Substance Abuse and Mental Health Services Administration (SAMHSA), UK National Health System (NHS), and Veterans Health Administration (VHA) promote integration of mental health into primary care (Figure $2 \mathrm{~B}$ ) ${ }^{25}$ This model is designed to address the high rate of general medical conditions reported in people with comorbid mental illness, the known decreased life expectancy of mentally ill patients, and stigma of visiting a mental health provider. ${ }^{26}$ However, patients with FND often present to subspecialists, such as neurologists or epilepsy specialists, and may pose significant difficulties for primary care and general mental health-care providers who do not have much experience in differentiating FND from neurological disorders. Another model of

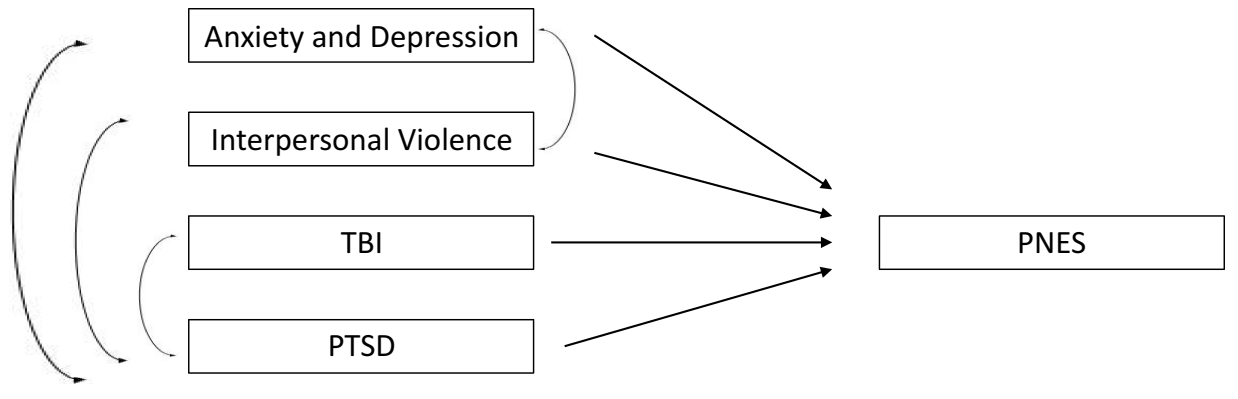

Figure I Path analysis of direct and indirect risk facts that may lead to PNES. 
A Center of Excellence

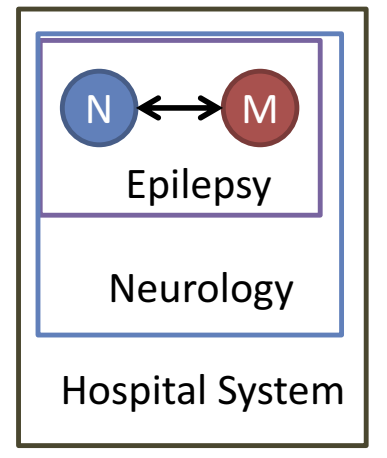

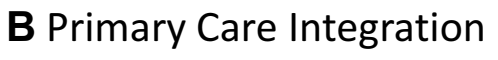

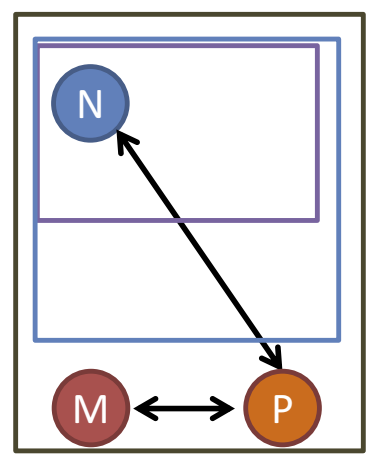

C Specialty Integration

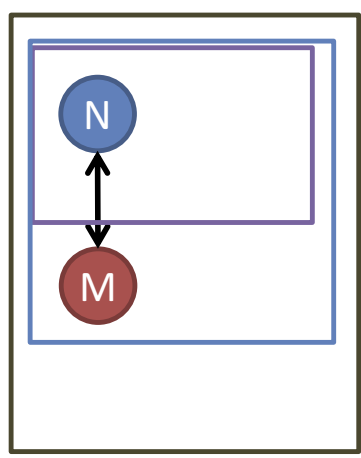

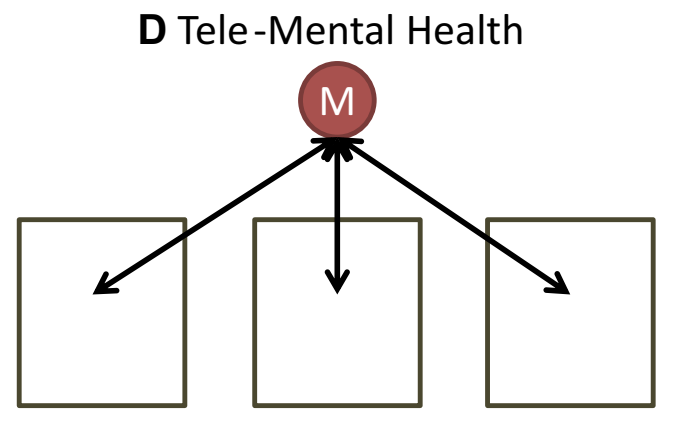

E MH Champion

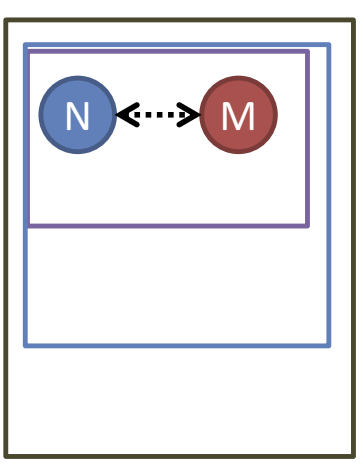

F Private Practice

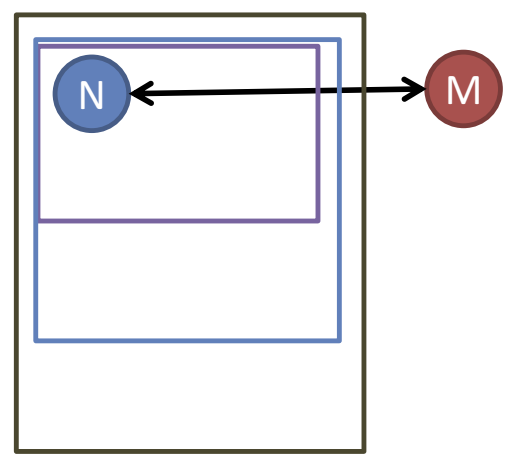

Figure 2 Models of Coordinating Neuro-Psych Care. (A) Center of Excellence Model hires a mental health provider within the epilepsy program; (B) mental health integration into primary care, this is the most popular model; (C) mental health is sometimes formally integrated in neurology at a departmental level; (D) tele-mental health models allow for mental health professionals to provide services remotely; (E) Mental health providers may develop informal relationship and develop a professional interest in epilepsy care; (F) many neurologists refer to out of network mental health providers.

Abbreviations: N, Neurologist; M, Mental Health-Care Provider; P, Primary Care Provider.

integrating mental health occurs at the specialty department level, in which neurology hires and/or partners with a mental health provider to care for a variety of patients with neurological conditions, including PNES (Figure 2C). Due to the relatively low volume of veterans with PNES, compared to other conditions such as PTSD, substance disorders, and mood disorders, facilities have been slow to hire a designated therapist dedicated to caring for patients with PNES. Instead, the VA established a National Tele-Mental Health Program for Non-Epileptic Seizures that provides specialized psychotherapy services for veterans with PNES remotely (Figure 2D). ${ }^{27}$ The relative effectiveness of this approach compared to other modalities of care has not been studied. Other epilepsy centers have informal relationships with mental health providers (Figure 2E). Finally, due to distance to VA facilities or gaps in care, some neurologists refer to care outside the VA system (Figure 2F). This study will examine how different models of care coordination affect seizure outcomes and utilization of health services: emergency room visits, hospital admissions, and the utilization of psychotherapy. ${ }^{28}$

\section{Methods/Design}

We hypothesize that the prevalence and incidence of veterans with PNES have increased from fiscal year 2002 to 2018 in post 9/11 veterans served by the VA, especially after the implementation of the Epilepsy Centers of Excellence (ECOE) in the VA in 2012. We also hypothesize that veterans with PNES, compared to veterans with a diagnosis of epilepsy or veterans with neither epilepsy nor PNES, will be more likely to be women, as well as have diagnoses of PTSD, depression, anxiety, and military sexual trauma (MST). Veterans with PNES developing after TBI (post-traumatic PNES or PTPNES) will have higher odds of being men, and having diagnoses of PTSD, depression, anxiety, MST, substance use disorder, and chronic pain, when compared to veterans with PNES without a history of TBI. Veterans who received treatment for PTSD, mood 
and anxiety disorders will have a lower risk of recurrent episodes of PNES, compared to those who did not receive these treatments. We also expect those with PNES who received treatment for PTSD, mood and anxiety disorders will have a lower average number of monthly psychogenic seizures and will more likely achieve psychogenic seizure freedom than those who did not receive treatment. Data accessed will be de-identified.

Objective 1: Describe the change in prevalence and incidence of veterans with post-traumatic and nontraumatic PNES in the VA Post 9/11 population over time.

Objective 2: Describe the risk factors (Female gender, PTSD, depression, anxiety, MST, chronic pain, TBI) for PNES including PNES subsequent to TBI (PTPNES).

Objective 3a: Explore the association between prior psychotropic and psychotherapeutic treatments for PTSD, mood and anxiety disorders, and the risk of developing PNES.

Objective 3b: Explore whether prior psychotropic and psychotherapeutic treatments for PTSD, mood and anxiety disorders are associated with the severity of PNES (number of monthly psychogenic seizures) and the likelihood of recovery (seizure-free for six months).

(Exploratory) Objective 4: Determine if change in PNES outcomes (as measured by seizure freedom or greater than $50 \%$ decrease in monthly seizures) is associated with the model of mental health-care coordination.

\section{Study Design Overview}

This is a retrospective cohort study focusing on the risk factors, health-care service delivery, and outcomes of post 9/11 veterans diagnosed with PNES. We will describe veterans' clinical and demographic characteristics, treatments, outcomes of epilepsy and PNES, and the impact of comorbid conditions on those outcomes.

\section{Study Population}

Researchers identified Post-9/11 veterans who received VA care for at least 3 years; groups will be divided into veterans with an established diagnosis of Epilepsy or PNES, and a comparison group who did not meet criteria for Epilepsy or PNES and are not in the process of being worked up for epilepsy or PNES (Figure 3). Since there is no ICD-9 code for PNES, we will deploy an informatics tool previously validated to identify PNES. ${ }^{29}$ This natural language processing classifier for the VA electronic health record correctly distinguished Post 9/11 veterans with epilepsy from veterans with PNES with $96 \%$ accuracy. ${ }^{29}$ The VA started the use of ICD-10 codes on October 1, 2015; therefore, cases of PNES beyond that time will be identified using ICD-10 code F44.5 (Conversion disorder with seizures or convulsions). Diagnostic levels of PNES certainty will be assigned using the International League Against Epilepsy (ILAE) criteria. ${ }^{8}$ The following table demonstrates the inclusion and exclusion criteria for the selection of PNES patients for this study (Table 1).

\section{Data Sources}

Data for this study will come from the VA and the US Department of Defense (DoD). Tables 2 and 3 provide brief descriptions of VA and DoD data sources used throughout the study. Data consistent across DoD and VA sources include ICD-9-CM and ICD-10-CM codes (schizophrenia [295.x, excluding 295.5 and F20.81, F20.89, F20.0, F20.1, F20.2, F20.9, F25.9], bipolar disorder [296.0-296.1, 296.4-296.8, and F30.10, F30.11, F30.12, F30.13, F30.2, F30.3, F30.4, F31, F32.9], other psychosis [297-298, and F22, F23, F24, F28, F29], depression [296.2-296.3, 311, and F32, F33], anxiety [300.00, 300.02, 300.09, and F41.9], PTSD [309.81 and F43.1], dementia [290.XX, 291.2, 294.XX, 331.XX, 046.1 and 046.3 , and F03, F05], traumatic brain injury [850, 800, 801, 803, 850, and 851-854, and Z87.820, S02.0X, S02.1X, S02.9X, S06.0X, S06.2X, S06.5X, S06.3, S06.4], delirium [293.0, 293.1, 292.0, 292.81, 291.0, 290.3, 290.41, 290.11 and F01.51, F03.90, F05, F06, F10.231, F11.921, F12.921, F13.921, F14.921, F15.921, F16.921, F18.921, F19.921, F19.939], mild cognitive impairment [331.83 and G31.84], and substance abuse [291, 292, 303-305, excluding 305.1, and F55, F10.159, F10.229, F10.231, F10.239, F10.27, F10.9, F10.27, F11.2, F12.10, F13.10, F14.10, F15.10, F16.10, F19.10, F19.2, F19.939] and medications for depression, anxiety, PTSD as well as those required to identify TBI severity, type of clinic in which care is received (e.g., neurology, primary care, and mental health), and demographic characteristics (e.g., age, sex, race/ethnicity, rank, component, branch of service, etc.). VA fee-based care will be examined to determine community (outside VA) care.

For Objective 1, we will use the Department of Defense (DoD) roster for demographic and military service data from Operations Enduring Freedom (OEF), Iraqi Freedom (OIF), and New Dawn (OND) data.

For Objectives 2 and 3, the DoD trauma registry will be accessed for medical trauma records by ICD-9 codes as well as Military Health System Data for inpatient, outpatient, and pharmacy data and will be linked by social security number to the Corporate Data Warehouse (CDW) data. The VA CDW file contains demographic, medical, psychiatric, medication and health-care utilization data. 


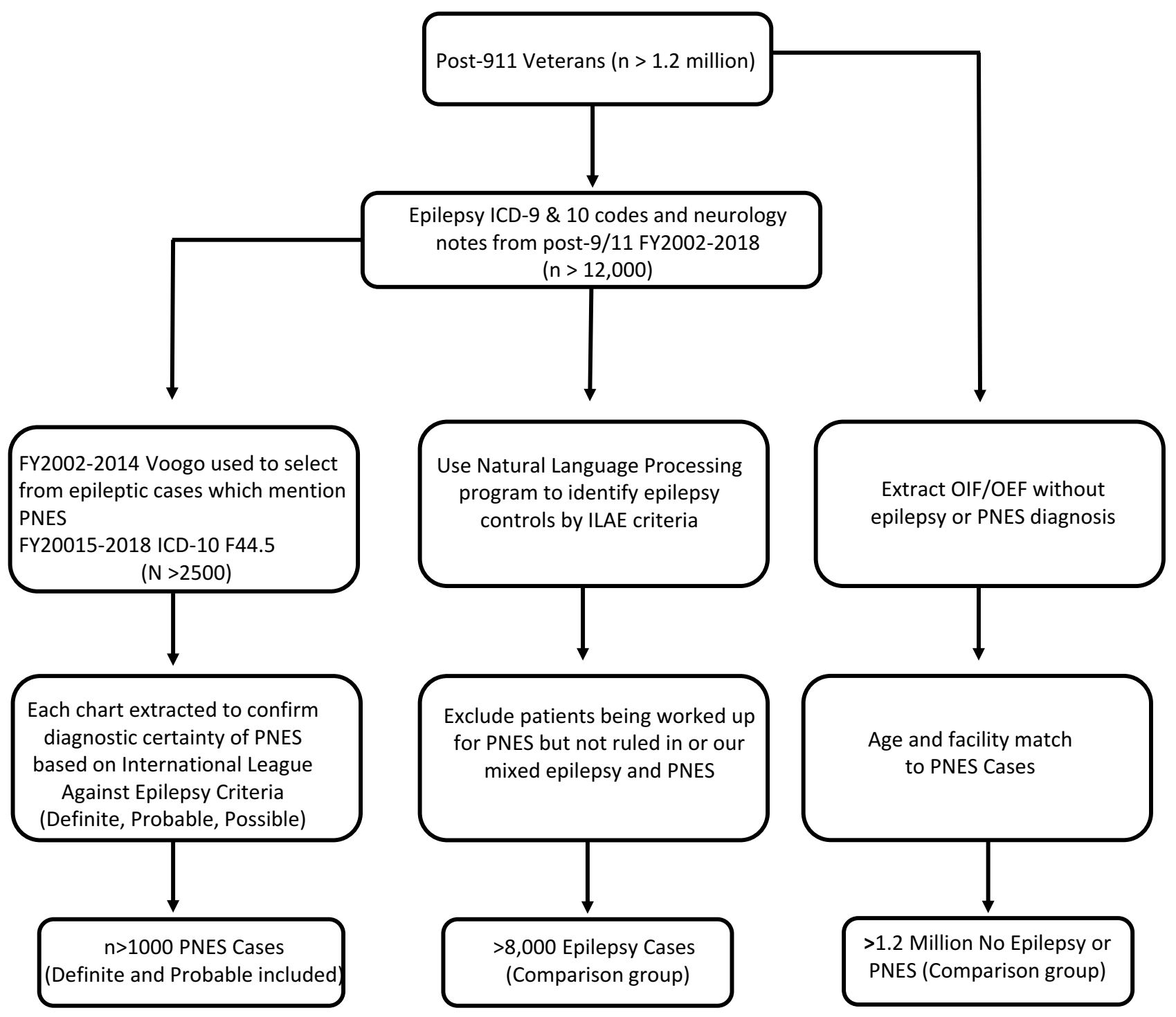

Figure 3 Selection of participants from the national VA database.

\section{Outcome Measures}

- PNES: PNES will be classified as newly diagnosed based on the index date of the first seizure, which is defined as the date of the first seizure documented in either the DoD or VA medical record.

- PNES classes: Based on the ILAE criteria, Definite PNES will have been diagnosed using VEEG monitoring, during which the veteran had a usual event without any concurrent changes in EEG. Probable PNES is diagnosed when a physician, with a working knowledge of PNES, observes the veteran during one of their spells or when PNES was documented to be diagnosed by VEEG by another institution but the report is not available. Possible PNES is diagnosed when the description of the events fits PNES.

PTPNES: will include individuals with a diagnosis of TBI in DoD or VA medical records prior to data of the first diagnosed seizure.

Inpatient procedures for epilepsy or seizures, including VEEG, will be defined from Current Procedural Terminology (CPT) codes. Non-VA service paid for by VA will be assessed using fee-for-services data.

Identifying Comorbidities: The presence of psychiatric conditions (i.e., PTSD, anxiety, depression, substance use disorders), with the exception of PNES, will be based on 
Table I Inclusion and Exclusion Criteria for the Selection of PNES Patients

\begin{tabular}{|l|l|}
\hline Inclusion Criteria & Exclusion Criteria \\
\hline Post-9/II veterans & No documented EEG \\
\hline $\begin{array}{l}\text { Enrolled in the VA with at least } 3 \text { years of care (at least one healthcare visit } \\
\text { each year for } 3 \text { years) }\end{array}$ & PNES was not documented as a differential diagnosis \\
\hline $\begin{array}{l}\text { ICD-9 (informatics tool to identify as there is no code) and ICD-I0 (F44.5) } \\
\text { diagnostic coding }\end{array}$ & $\begin{array}{l}\text { Seizures due to physiological problem (alcohol withdrawal, } \\
\text { syncope, substance abuse) }\end{array}$ \\
\hline $\begin{array}{l}\text { Documentation of the following: pseudo-seizures, psychogenic seizures, stress- } \\
\text { related seizures, anxiety-related seizures }\end{array}$ & \\
\hline
\end{tabular}

Table 2 Description of Data Sources

\begin{tabular}{|l|l|}
\hline Data Sources & Description \\
\hline VA/DOD OEF/OIF/OND Roster & $\begin{array}{l}\text { This file contains demographic and military service data for all service members deployed in support of OEF/ } \\
\text { OIF/OND including information regarding the first deployment start and end dates and last deployment start } \\
\text { and end dates. }\end{array}$ \\
\hline VA CDW & See Table 3 for specifics (clinical and administrative health care data) \\
\hline $\begin{array}{l}\text { TBI Screen and Comprehensive } \\
\text { TBI Evaluation }\end{array}$ & Contained in CDW files \\
\hline $\begin{array}{l}\text { DoD Military Health System Data } \\
\text { Repository }\end{array}$ & $\begin{array}{l}\text { We will obtain the Military Health System Data repository. Data includes inpatient, outpatient, and pharmacy } \\
\text { data similar to those in the VA and can be linked by social security number. }\end{array}$ \\
\hline DoD Trauma Registry (DoDTR) & $\begin{array}{l}\text { DoD database containing medical trauma record data elements (e.g., mechanism of injury, ICD-9-CM codes, } \\
\text { Abbreviated Injury Score, CPT4 codes, etc.) from military levels of care I (first responder) through level } \\
\text { V (definitive care both in and outside the continental United States). }\end{array}$ \\
\hline
\end{tabular}

Abbreviations: VA, Veterans Affairs; DOD, Department of Defense; OEF, Operation Enduring Freedom; OIF, Operation Iraqi Freedom; OND, Operation New Dawn; TBI, Traumatic Brain Injury; CDW, Corporate Data Warehouse; CPT, Current Procedural Terminology; ICD, International Classifications of Diseases.

ICD-9 codes in both DoD and VA data. The addition of DoD data will improve the ascertainment and temporal accuracy of exposures and comorbid diagnoses (including TBI and PTSD). TBI and PTSD severity will be identified in VA and DoD data using the algorithm developed by the Armed Forces Health Surveillance. We will calculate the index date for TBI diagnosis as the first diagnosis of any TBI.

Services provided for mental health within the VA mental health encounters will be defined using CPT for nonpharmacologic mental health treatment (psychotherapy and health \& behavior intervention): 90832; 90834; 90837; 90847; 90853; 96152; 96153; 96154. Neurology visits will be captured by VA stop codes 345 for Epilepsy Center of Excellence and 314 for General Neurology. Non-VA service paid for by VA will be assessed using fee-for-services data.

MST has been defined by the VA (VA; 2004) as "sexual harassment that is threatening in character or physical assault of a sexual nature that occurred while the victim was in the military, regardless of geographic location of the trauma, gender of victim, or the relationship to the perpetrator." The VA has an MST screener, which is asked of all veterans during clinical appointments.

Pain and Chronic Pain: We will use the vital sign pain intensity numeric rating scale (NRS) score to determine the level of pain reporting. For the NRS, veterans are asked "on a scale of 0 to 10 , where 0 means no pain and 10 equals the worst possible pain, what is your current pain level?" We will categorize the NRS into four levels: 0 as no pain; 1 to 3 as mild; 4 to 6 as moderate; and 7 or greater as severe pain. ${ }^{30}$ We chose these thresholds because the VA's "Pain as the 5th Vital Sign" initiative states that "it is reasonable to expect that a pain score of 4 or higher would trigger a comprehensive pain assessment and prompt intervention" by the provider. ${ }^{31}$ Chronic pain will be defined as 3 or more pain scores of 4 or higher within 90 days. If there are more than one NRS recorded in the EHR on a day, we will retain the highest one. 
Table 3 Further Details on the Data from the VA's Corporate Data Warehouse Files

\begin{tabular}{|c|c|}
\hline Variables & Source(s) \\
\hline $\begin{array}{l}\text { Demographics: Date of Birth (DOB), race/ethnicity, sex, home zip code, } \\
\text { service connected status }\end{array}$ & Corporate data warehouse (CDW) Patient files \\
\hline $\begin{array}{l}\text { Medical and psychiatric conditions: muscular skeletal disorders (MSD), } \\
\text { Post traumatic Stress Disorder (PTSD), depression, substance use } \\
\text { disorder, etc. }\end{array}$ & CDW Visit files \\
\hline Consults, referrals & CDW consults \\
\hline Visits, utilization & CDW/Decision Support Services (DSS) - fee basis, utilization, stop codes \\
\hline Medications & $\begin{array}{l}\text { CDW - Pharmacy files (RXOutpatFill, RxOutpat, RxOutpatSig), Non-VA } \\
\text { Meds }\end{array}$ \\
\hline Station, VISN facility size, geographic location & CDW - Patient files \\
\hline Smoking, alcohol, PTSD, and depression screen Results & CDW - Health Factors, Mental Health, psych instruments \\
\hline Progress notes, primary care provider Information & CDW - primary care management module (PCMM), progress notes \\
\hline Non-VA care & Fee basis, VA Choice tables \\
\hline Pain scores & Vital Signs \\
\hline
\end{tabular}

Abbreviations: CDW, Corporate Data Warehouse; VISN, Veterans Integrated Services Networks; PTSD, Post-Traumatic Stress Disorder; VA, Veterans Affairs.

Mental Health Diagnoses: Individuals with at least two outpatient visits or one inpatient visit with the ICD-9 codes indicative of PTSD, depression, anxiety, or bipolar disorder in DoD or VA data will be identified as having that condition.

\section{Study Status}

Medical record review began in August 2018. A total of 736 records were identified: 414 veterans with a definitive (VEEG) diagnosis of PNES, 114 veterans with a probable diagnosis, and 208 with a possible diagnosis. Demographic, clinical, treatment, and outcomes data for epilepsy and PNES patients have been collected. The next phase will involve locating individuals with an ICD-10 diagnosis of PNES and proceed with data collection.

\section{Statistical Analysis \\ Objective I}

Describe the change in prevalence and newly diagnosed veterans with post-traumatic and non-traumatic PNES in the VA Post $9 / 11$ population. Researchers primary analysis will use Generalized Estimating Equations (GEE) to determine if the rate of change in newly diagnosed PNES increases over time; these models will then be stratified by TBI exposure to explore whether TBI is significantly associated with that change. We will also explore whether changes in the sex and age distributions of veterans entering the VA over time are related to change in PNES through stratification of these models. The outcome distribution will be Poisson and a log-link will be used. Secondary analysis will estimate the number of newly diagnosed veterans and prevalence rates of PNES using Poisson regression. The measure will be rate per entry year into the VA (regardless of year of PNES). As noted above, TBI, age, and sexstratification of models will be conducted in order to better understand the inter-relationships of these variables with PNES over time. Secondary results will be compared to the primary analysis in order to explore different questions regarding the magnitude of PNES reveal and aspects of its presentation. Since the outcome of interest is a count variable and we wish to model the rate of seizures, we will use Poisson or zero-inflated Poisson models. A Poisson regression power analysis module will be used to calculate the power to detect a $20 \%$ increase in the PNES rate per year, starting with a rate in the baseline year of 3 per 100,000 people per year. $^{32}$

\section{Objective 2}

Describe the risk factors (female sex, PTSD, Depression, Anxiety, MST, Chronic Pain, mTBI) for PNES including PNES subsequent to TBI (PTPNES). Two logistic regressions will be conducted: 1) Binary outcome is Epilepsy/ PNES; and 2) PNES/No PNES (epilepsy cases removed). The covariates in these 2 models will be: any prior history of 
TBI, PTSD, depression, anxiety, substance use disorder, and military sexual trauma, adjusting for confounders such as age, sex, race/ethnicity, and marital status. In addition, crosslagged models, a type of structural equation model (specifically a path analysis model), will be used to look at direct and indirect associations between TBI, PTSD, and PNES over time. Covariates will be the same clinical and demographic characteristics as used in the logistic regressions above. Standardized path coefficients will be used to control for difference in variance between constructs. Path coefficients with $p<0.01$ will be considered statistically significant. Since the outcome of interest is comparison of risk factors (sex, PTSD, depression), the temporal sequence of the relative rate will be used, while risk factors that occur concurrently will be analyzed using logistic regression.

\section{Objective 3}

Explore whether prior psychotropic and psychotherapeutic treatments for mood and anxiety disorders decrease the risk of developing PNES. Time-to-event models will be used to examine how pharmacologic treatments for PTSD (or comorbid depression or anxiety) are associated with PNES development and seizure resolution. Treatments will be entered into these models as time-varying covariates based on treatment start and stop dates as well as clinical knowledge of their effects (e.g., 30 days on psychotropic medications, time until completion of CBT, half-life of medication). Presence of comorbid depression and anxiety as well as demographic variables will be examined as mediators and moderators of the treatment-outcome association. Time will start at entry into the cohort by 1st VA visit and finish with either the date of diagnosis of PNES (PNES development) or data when file notes patient is seizure-free (Seizure resolution), or completion of the follow-up period (September 30, 2018, or death). Additionally, researchers may explore the impact of mental health services using same model of objective 3, with therapy being a time-varying covariate as above. Models will integrate both pharmacologic and non-pharmacologic interventions as above to determine if there is any cumulative or interactive effect. We will analyze number of psychogenic seizures pre-post mental health intervention using ANCOVA. The post-treatment severity score will be considered the outcome while treatment (either pharmacologic or psychotherapy) is dichotomous and pre-treatment (or baseline seizure severity) is controlled for by adding the variable to the model. As with any administrative data, it is likely that some data will be inconsistent or missing.
Our preliminary analyses indicate that for most key variables, less than $3 \%$ of data are missing. In particular, NRS are missing few recent visits. We will flag records that have discrepancies and conduct a stratified analysis as part of the sensitivity analyses. Sensitivity analyses using multiple imputation under missing not at random assumptions will be performed to examine the robustness of conclusions of the primary analysis to missing data. ${ }^{31-34}$ Since the outcome of interest is time to event, we will use survival methods.

\section{Discussion}

With increasing incidence and prevalence of PNES, it is important to evaluate the effectiveness of integrated mental health. Through the VA system, we will be able to evaluate the effectiveness of an integrated healthcare system in a model that resembles a naturalistic observational cohort study. Studies using the VA EHR have been used in other conditions such as HIV/AIDS. Using the national VA EHR, we can take advantage of the granular individual clinical data as well as the variety of care models across different facilities. The results will have immediate and practical application in health-care operations and epilepsy programming. If effectiveness of specific care models is demonstrated, this will support the implementation of these services at a larger scale throughout the ECOEs. We will use the data to examine barriers to care and identify which regions and facilities have more difficulty with access to care.

Definitive treatment for PNES has not been established. Through the VA, there is the possibility to look at continuity of care, as well as implement an active monitoring system using a clinical cohort to facilitate clinical trials for PNES. Qualitative studies can also be conducted to provide further insight on the providers' perspective of the effectiveness of care implementation. Multiple outcome measurements will be included in this study. In addition to seizure frequency, we will include emergency room visits, hospital admissions, and psychotherapy utilization. These measures will be collected prior to and after the diagnosis of PNES. Seizure frequency will be measured at 6 months, 1, 2, and 5 year follow-up. Fluctuations in seizure frequency can be recorded over the cohort, and the effect of interventions, such as psychotherapy, can be looked at in relation to these fluctuations in seizure frequency. Following this cohort for up to 5 years allows us to look at long-term efficacy of interventions in PNES. 
Much of the current discourse on PNES argues that it is either caused by or another manifestation of PTSD, particularly within the veteran population. However, our clinical experience and hypothesis is that many of our patients with PTSD have already received extensive treatment for PTSD prior to developing PNES, which could be an important confounding factor if not a risk factor. Multiple other risk factors have been identified in PNES, and unfortunately, patients with FNDs often report more than one. By describing the complex precipitants and risk factors to PNES, we may shift how PNES is conceptualized. Perhaps it is a condition that results from psychological trauma but is not responsive to mainstay PTSD treatment. Another possibility is that some conditions make treatment more efficacious or some models of care are more effective for veterans with PNES. An improved understanding of the interplay of these factors could help tailor therapy to individual patients. In addition to studying clinical efficacy of psychotropic and psychotherapeutic interventions, we can study the effectiveness of the telemental health model, used specifically at the VA. Education efforts may clarify the diagnostic process and the inappropriate prescription of AEDs, and could facilitate timelier referral for VEEG; these results could be part of the intermediate benefits of this study. Future research such as developing genetic, proteomic, neuroimaging, neurophysiological and other advanced biomarkers will be dependent on how well we characterize PNES phenotypes. Past research has been limited to characterizing more severe and refractory people with PNES and our study will better characterize a more representative population.

\section{Strengths}

Our study represents a unique opportunity to draw from national data and observe the course of PNES over time. While case identification and outcomes rely on data extraction from the EHR, the data set approximates a population level study of PNES. This large cohort provides a rich data set to examine multiple, complex risk factors and their temporal relationships. The VA is among the few sources that provide large, nationally representative, and granular clinical data. In this study, we will explore multiple outcome measurements such as healthcare utilization in emergency room visits and hospital admissions, as well as mental health service utilization in the type of therapy, number of visits with the provider, and completion of therapy.

\section{Limitations}

Among the limitations of the study is the identification of PNES. Since many veterans are diagnosed in non-VA facilities, we did not want to limit our sample to only those who had VEEG done within the VA or DoD. Instead, we searched for notes that would communicate how the diagnosis of PNES was made. An alternative approach would be to explore risk factors and outcomes only in veterans who have documented VEEG reports diagnosing PNES within the VA EHR. This would decrease our power significantly, but we will explore whether there are significant differences between those veterans diagnosed with PNES with and without clearly documented VEEG reports in the VA EHR. Another inherent limitation to any PNES study is that since the definitive diagnosis is made by VEEG, veterans who do not have access to VEEG, do not follow-up with VEEG, or are not referred to VEEG will not have a definitive diagnosis. Another potential limitation is the military members and veterans who do not follow up within the VA or who receive care outside the VA will not be identified since the data depends on the VA EHR. This may underestimate the incidence and burden of PNES. Finally, selection bias may occur due to the VA eligibility (which is based on lower socioeconomic status), people with PNES tend to be higher health-care users, and veterans tend to be disproportionally male compared to the civilian population. Our results still have external generalizability since people with PNES tend to be of lower socioeconomic status and high health-care users.

\section{Conclusion}

PNES represents a model FND with which to study integration of care. PNES patients receive the greatest benefit from a multidisciplinary team comprised neurologists, epileptologists, psychologists, and primary care providers. In this study, we will use multiple outcome measures such as psychogenic seizure burden and healthcare service utilization to assess the success of an integrated care model. Our study will provide guidance for the treatment of PNES within the VA's integrated care model, as well as a prototype for future virtual cohort studies of integrated care management of complex disorders.

\section{Abbreviations}

ACT, Acceptance and Commitment Therapy; CBT, Cognitive Behavioral Therapy; CDW, Corporate Data Warehouse; CPT, Current Procedural Terminology; DoD, Department of 
Defense; ECOE, Epilepsy Centers of Excellence; EHR, Electronic Health Records; FND, Functional Neurological Disorder; GEE, Generalized Estimating Equations; ICD, International Classification of Disease; ILAE, International League Against Epilepsy; MST, Military Sexual Trauma; NRS, Numeric Rating Scale; OEF, Operation Enduring Freedom; OIF, Operation Iraqi Freedom; OND, Operation New Dawn; PE, Prolonged Exposure; PNES, Psychogenic Non-Epileptic Seizures; PTPNES, Post-Traumatic PNES; PTSD, Post-Traumatic Stress Disorder; SSRI, Selective Serotonin Reuptake Inhibitor; TBI, Traumatic Brain Injury; VA, Veterans Affairs; VEEG, Video-Electroencephalogram.

\section{Ethics Approval and Consent to Participate}

Ethical approval was granted by the VA Connecticut Healthcare System Institutional Review Board and by the US Army Medical Research Human Research Protection Office.

\section{Data Sharing Statement}

The datasets generated and/or analyzed during the current study are not publicly available due to VA privacy and information security policies.

\section{Acknowledgments}

We would like to thank members of the VA Connecticut Healthcare System Pain, Research, Informatics, Medical Comorbidities and Education Center of Innovation and the Yale Center for Neuroepidemiology and Clinical Neurological Research for their input and feedback.

\section{Author Contributions}

$\mathrm{HA}, \mathrm{BF}, \mathrm{MJP}$ designed the project. HA secured funding for the project and was responsible for ethics approval. All authors contributed to the conception and design of the study, interpretation of data, draft composition and review, gave final approval of the version to be published, and agreed to be accountable for all aspects of the work.

\section{Funding}

The study is funded through the US Department of Defense Congressionally Directed Medical Research Programs (EP1600449).

\section{Disclosure}

Dr Daniela Galluzzo reports grants from Department of Defense, during the conduct of the study. Dr Yarden
Bornovski reports grants from Congressionally Directed Medical Research Programs, during the conduct of the study. Dr Benjamin Tolchin reports grants from US Department of Veterans Affairs and CG Swebilius Trust, outside the submitted work. Dr Mary Jo Pugh reports grants from Brain Sentinal, outside the submitted work. Dr. Hamada Altalib has received research funding from Sunovion, Eisai, UCB, and Pfizer. Dr. Altalib has also served on the advisory board for Eisai. The authors report no other conflicts of interest in this work.

\section{References}

1. Saxe GNC, Berkowitz R, Hall K, Lieberg G, Schwartz J, van der Kolk B. A Somatization in Patients with Dissociative Disorders. Am J Psychiatry. 1994;151:1329-1334.

2. Binder LS, Salinsky MC. Psychogenic nonepileptic seizures. Neuropsychol Rev. 2007;17:405-412. doi:10.1007/s11065-007-9047-5

3. Chen DS, LaFrance E. Psychogenic non-epileptic seizures. Current Neurol Neurosci Reposit. 2017;17:70-80.

4. Gasparini SB, Ferlazzo E, Beghi E, et al. Management of psychogenic non-epileptic seizures: a multidisciplinary approach. Eur $J$ Neurol. 2018;26:205-213. doi:10.1111/ene.13818

5. LaFrance WC Jr, Reuber M, Goldstein L. Management of psychogenic nonepileptic seizures. Epilepsia. 2013;54(1):53-67. doi:10.1111/ epi.12106

6. Voon V, Cavanna AE, Coburn K, Sampson S, Reeve A, LaFrance WC Jr. Functional neuroanatomy and neurophysiology of functional neurological disorders (Conversion Disorder). J Neuropsychiatry Clin Neurosci. 2016;28(3):168-190. doi:10.1176/ appi.neuropsych.14090217

7. Kerr WT, Janio EA, Le JM, et al. Diagnostic delay in psychogenic seizures and the association with anti-seizure medication trials. Seizure. 2016;40:123-126. doi:10.1016/j.seizure.2016.06.015

8. LaFrance WC Jr, Baker GA, Duncan R, Goldstein LH, Reuber M. Minimum requirements for the diagnosis of psychogenic nonepileptic seizures: a staged approach: a report from the International League Against Epilepsy Nonepileptic Seizures Task Force. Epilepsia. 2013;54(11):2005-2018. doi:10.1111/epi.12356

9. Reuber M. The etiology of psychogenic non-epileptic seizures: toward a biopsychosocial model. Neurol Clin. 2009;27(4):909-924. doi:10.1016/j.ncl.2009.06.004

10. Salinsky M, Rutecki P, Parko K, et al. Psychiatric comorbidity and traumatic brain injury attribution in patients with psychogenic nonepileptic or epileptic seizures: a multicenter study of US veterans. Epilepsia. 2018;59(10):1945-1953. doi:10.1111/epi.14542

11. Salinsky M, Evrard C, Storzbach D, Pugh MJ. Psychiatric comorbidity in veterans with psychogenic seizures. Epilepsy Behav. 2012;25 (3):345-349. doi:10.1016/j.yebeh.2012.07.013

12. Casey JA, Schwartz BS, Stewart WF, Adler NE. Using electronic health records for population health research: a review of methods and applications. Annu Rev Public Health. 2016;37:61-81.

13. LaFrance CWB, Barry GL, Blum JJ, et al. Multicenter pilot treatment for psychogenic nonepileptic seizures a randomize clinical trial. $J \mathrm{Am}$ Med Assoc. 2014;71(9):997-1005.

14. Goldstein LHM, Landau JDC, Stone S, et al. Cognitive behavioural therapy vs standardised medical care for adults with Dissociative non-Epileptic seizures (CODES): a multicentre randomised controlled trial protocol. BMC Neurol. 2015;15(98):1-13. doi:10.1186/s12883-0150350-0

15. Allen LW, Escobar R, Gara J, Hamer MR. Cognitive-Behavioral Therapy for Somatization Disorder. Am Med Assoc. 2006;166:1512-1518. 
16. Tolchin B, Baslet G, Suzuki J, et al. Randomized controlled trial of motivational interviewing for psychogenic nonepileptic seizures. Epilepsia. 2019;60(5):986-995. doi:10.1111/epi.14728

17. Goldstein LH, Chalder T, Chigwedere C, et al. Cognitive-behavioral therapy for psychogenic nonepileptic seizures: a pilot RCT. Neurology. 2010;74(24):1986-1994. doi:10.1212/WNL.0b013e3181e39658

18. Barry JJ, Wittenberg D, Bullock KD, Michaels JB, Classen CC, Fisher RS. Group therapy for patients with psychogenic nonepileptic seizures: a pilot study. Epilepsy behav. 2008;13(4):624-629. doi:10.1016/j.yebeh.2008.06.013

19. Mayor R, Howlett S, Grunewald R, Reuber M. Long-term outcome of brief augmented psychodynamic interpersonal therapy for psychogenic nonepileptic seizures: seizure control and health care utilization. Epilepsia. 2010;51(7):1169-1176. doi:10.1111/(ISSN) 1528-1167

20. Graham CD, O'Hara DJ, Kemp S. A case series of Acceptance and Commitment Therapy (ACT) for reducing symptom interference in functional neurological disorders. Clin Psychol Psychother. 2018;25 (3):489-496. doi:10.1002/cpp.v25.3

21. Baslet G, Dworetzky B, Perez DL, Oser M. Treatment of psychogenic nonepileptic seizures: updated review and findings from a mindfulness-based intervention case series. Clin EEG Neurosci. 2015;46(1):54-64. doi:10.1177/1550059414557025

22. LaFrance WC Jr, Miller IW, Ryan CE, et al. Cognitive behavioral therapy for psychogenic nonepileptic seizures. Epilepsy Behav. 2009;14(4):591-596. doi:10.1016/j.yebeh.2009.02.016

23. Goldstein LH, Mellers JD, Landau S, et al. Cognitive behavioural therapy vs standardised medical care for adults with Dissociative non-Epileptic Seizures (CODES): a multicentre randomised controlled trial protocol. BMC Neurol. 2015;15:98.

24. McMillan KK, Pugh MJ, Hamid H, et al. Providers' perspectives on treating psychogenic nonepileptic seizures: frustration and hope. Epilepsy Behav. 2014;37:276-281. doi:10.1016/j.yebeh.2014.07.001
25. Kanemoto K, LaFrance WC Jr, Duncan R, et al. PNES around the world: where we are now and how we can close the diagnosis and treatment gaps-an ILAE PNES Task Force report. Epilepsia Open. 2017;2(3):307-316. doi:10.1002/epi4.12060

26. Ahmedani BK, Osborne J, Nerenz DR, et al. Diagnosis, costs, and utilization for psychogenic non-epileptic seizures in a US health care setting. Psychosomatics. 2013;54(1):28-34. doi:10.1016/j. psym.2012.08.005

27. Darkins A. The growth of telehealth services in the Veterans Health Administration between 1994 and 2014: a study in the diffusion of innovation. Telemed J E Health. 2014;20(9):761-768. doi:10.1089/ tmj.2014.0143

28. Levin BL, Petrila J, Hennessy KD. Mental Health Services: A Public Health Perspective. USA: Oxford University, ; 2004.

29. Hamid H, Fodeh SJ, Lizama AG, et al. Validating a natural language processing tool to exclude psychogenic nonepileptic seizures in electronic medical record-based epilepsy research. Epilepsy behav. 2013;29:578-580. doi:10.1016/j.yebeh.2013.09.025

30. Krebs EE, Carey TS, Weinberger M. Accuracy of the pain numeric rating scale as a screening test in primary care. J Gen Intern Med. 2007;22(10):1453-1458. doi:10.1007/s11606-007-0321-2

31. Mularski RA, White-Chu F, Overbay D, Miller L, Asch SM, Ganzini L. Measuring pain as the 5th vital sign does not improve quality of pain management. J Gen Intern Med. 2006;21(6):607-612. doi:10.1111/j.1525-1497.2006.00415.x

32. Asadi-Pooya AA, Sperling MR. Epidemiology of psychogenic nonepileptic seizures. Epilepsy Behavior. 2015;46:60-65. doi:10.1016/j. yebeh.2015.03.015

33. Molenberghs G, Thijs H, Jansen I, et al. Analyzing incomplete longitudinal clinical trial data. Biostatistics. 2004;5(3):445-464. doi:10.1093/ biostatistics/kxh001

34. Lachin JM. Statistical considerations in the intent-to-treat principle. Control Clin Trials. 2000;21(3):167-189. doi:10.1016/S0197-2456(00) 00046-5
Neuropsychiatric Disease and Treatment

\section{Publish your work in this journal}

Neuropsychiatric Disease and Treatment is an international, peerreviewed journal of clinical therapeutics and pharmacology focusing on concise rapid reporting of clinical or pre-clinical studies on a range of neuropsychiatric and neurological disorders. This journal is indexed on PubMed Central, the 'PsycINFO' database and CAS, and

\section{Dovepress}

is the official journal of The International Neuropsychiatric Association (INA). The manuscript management system is completely online and includes a very quick and fair peer-review system, which is all easy to use. Visit http://www.dovepress.com/testimonials.php to read real quotes from published authors. 